\title{
氧化物陶瓷闪烧机理及其应用研究进展
}

\author{
刘金铃 ${ }^{1}$, 刘佃光 ${ }^{2}$, 任 科 $^{3}$, 王一光 ${ }^{3}$
}

(1. 西南交通大学 力学与航空航天学院, 成都 611756; 2. 西南交通大学 材料科学与工程学院, 成都 610031;

3. 北京理工大学 先进结构技术研究院, 北京 100081)

摘 要: 闪烧是近些年广受关注的一种电场辅助烧结技术。本文介绍了闪烧的起源与发展, 并对闪烧的基本特征进 行了分析。在闪烧孕育与引发过程的研究方面, 发现了孕育阶段的非线性电导特征和电化学黑化现象, 提出了氧空 位主导的缺陷机制; 在闪烧阶段的快速致密化研究方面，提出了电场作用导致的缺陷产生和运动会在粉体颗粒间 产生库仑力，有利于烧结前期的致密化过程，同时发现闪烧致密化过程中还伴随着金属阳离子的快速运动; 在闪 烧阶段的晶粒生长和微结构演变方面, 发现了试样温度沿电流方向呈非对称分布, 试样中间位置的晶界迁移率明 显提高, 提出电化学缺陷对微观结构有重大影响。基于上述研究成果, 本团队利用电场作用下出现的低温快速传质 现象，发展了陶瓷闪焊技术，实现了同种陶瓷/陶瓷、陶瓷/金属，甚至异种陶瓷/陶瓷之间的快速连接; 发展了陶瓷 闪烧合成技术，不仅实现了典型氧化物陶瓷的快速合成，而且实现了高熵陶瓷和具有共晶形貌的氧化物陶瓷的快 速合成; 发展了氧化物陶瓷的电塑性成形技术, 初步实现了氧化锆陶瓷低温低应力下的快速拉伸和弯曲变形。本文 最后总结了闪烧机理研究面临的挑战，并从焦耳热效应和非焦耳热效应两方面展望了闪烧的发展方向，期望对闪 烧技术在国内的发展有所禆益。

关 键 词: 闪烧; 焦耳热; 缺陷; 连接; 合成; 成形; 综述

中图分类号: TQ174 文献标志码: A

\section{Research Progress on the Flash Sintering Mechanism of Oxide Ceramics and Its Application}

\author{
LIU Jinling $^{1}$, LIU Dianguang ${ }^{2}$, REN Ke $^{3}$, WANG Yiguang ${ }^{3}$
}

(1. School of Mechanics and Aerospace Engineering, Southwest Jiaotong University, Chengdu 611756, China; 2. School of Materials Science and Engineering, Southwest Jiaotong University, Chengdu 610031, China; 3. Institute of Advanced Structure Technology, Beijing Institute of Technology, Beijing 100081, China)

\begin{abstract}
Flash sintering is an electric field assisted sintering technology which has attracted much attention in recent years. This review introduces its origin, development, and basic characteristics. In the study of flash incubation and initiation process, the nonlinear conductivity characteristics and electrochemical blackening phenomenon are narrated, and the defect mechanism dominated by oxygen vacancy is recounted. As for rapid densification during flash sintering, it is proposed that the generation and movement of defects caused by electric field produce Coulomb force between powder particles, which is conducive to density in the early stage of flash sintering. Meanwhile, the
\end{abstract}

收稿日期：2021-08-19; 收到修改稿日期：2021-10-21; 网络出版日期：2021-10-21

基金项目：国家自然科学基金重点项目(51732009) National Natural Science Foundation of China (51732009)

作者简介：刘金铃(1983-), 男, 教授. E-mail: liujinling@swjtu.edu.cn LIU Jinling (1983-), male, professor. E-mail: liujinling@swjtu.edu.cn

通信作者: 王一光，教授. E-mail: wangyiguang@bit.edu.cn WANG Yiguang, professor. E-mail: wangyiguang@bit.edu.cn 
densification process is accompanied by the rapid movement of metal cations. In terms of grain growth and microstructure evolution during the flash sintering, the sample temperature is asymmetrically distributed along the current direction, and the internal grain boundary mobility in the sample is significantly improved. During this stage, electrochemical defects exert a significant impact on the microstructure. Based on the above researches, we developed ceramic flash joining technology by using phenomenon of low-temperature rapid mass transfer under electric field, and realized rapid joining between similar kind of ceramics/ceramics, ceramics/metals, and even dissimilar ceramics/ceramics. A new ultrafast ceramic synthesis technology by flash sintering was developed, which not only realized the rapid synthesis of typical oxide ceramics, but also realized the rapid synthesis of high entropy ceramics and oxide ceramics with eutectic morphology. An electroplastic forming technology of oxide ceramics was developed, and a rapid tensile and bending deformation of zirconia ceramics at low temperature and low stress was preliminarily realized. Finally, this review summarizes the challenges in the field of flash sintering mechanism, and looks forward to the development direction of flash sintering from two aspects of Joule heating effect and nonthermal effect, aiming to be beneficial to the development of flash sintering technology in China.

Key words: flash sintering; Joule heating; defect; joining; synthesis; forming; review

电场和磁场对陶瓷致密化、晶粒生长和塑性变 形行为的影响已有较长研究历史。2010 年, 美国科 罗拉多大学 Rishi Raj 教授课题组意外发现: 在临界 直流电场 $(\geqslant 60 \mathrm{~V} / \mathrm{cm})$ 作用下, 氧化钇稳定氧化锆陶 瓷(3YSZ) 可以在 $850{ }^{\circ} \mathrm{C}$ 的炉温下实现烧结, 而且 试样的相对密度由 $50 \%$ 达到完全致密只需要几秒钟, 他们将这种新型烧结技术命名为 “闪烧 (Flash Sintering) ${ }^{\text {"[1] }}$, 由此掀起了继微波烧结和放电等离子 烧结之后新一轮场辅助烧结技术的研究热潮。随后 美国、意大利、德国、英国、巴西、法国和日本等 国的学者相继对闪烧开展了研究。国内北京理工大 学、西北工业大学、西南交通大学、武汉理工大学、 长安大学、郑州大学、上海交通大学和中国工程物 理研究院等单位也紧随其后展开了相关研究。2017 年，国家自然科学基金委支持了王一光教授申报的 “陶瓷材料的闪烧制备新技术及机理研究”重点项目, 旨在探索闪烧过程中产生非线性电导、快速致密化 和微结构演化的内在机理, 发展基于闪烧原理的陶 瓷制备新技术。

目前, 已有多篇综述论文对闪烧的研究现状进 行了总结 ${ }^{[2-7]}$, 对闪烧的工艺特点、实验方法和材料 体系进行了介绍, 对基于焦耳热、Frenkel 缺陷和电 化学缺陷的闪烧机理进行了归纳分析。本文主要围 绕近年来本团队在氧化物陶瓷闪烧领域的研究成果, 阶段性总结氧化物陶瓷闪烧微观机理和闪烧制备新 技术方面的研究进展, 并对未来的发展方向进行展 望, 希望为国内闪烧技术的发展提供参考。

\section{1 闪烧过程及其特点}

如图 1 所示, 在置于加热装置内的狗骨状生坏 试样两端连接上电极, 再将电极与外部电源相连, 就构成了一套简单的闪烧实验装置。也有研究者在 闪烧实验中使用条状、棒状或圆柱状试样 ${ }^{[7]}$ 。陶瓷 生坏在合适的温度和电场条件下就能发生闪烧。常 用管式炉或箱式炉加热生坏来达到发生闪烧所需的 起始温度。对于能够在低温下发生闪烧的样品, 可 用加热板或加热套来替代高温炉。如果样品在室温 下即能发生闪烧, 就可以完全去掉加热装置。外部 电源为生坏发生闪烧提供所需的起始电压, 常用的 设备是直流电源, 闪烧所需的电场强度一般从几伏/ 毫米到几百伏/毫米, 远低于电火花放电(Electric Discharge)或电弧放电(Arc Discharge)烧结的电场强 度( 几千伏/毫米到几万伏/毫米), 又明显高于放电 等离子烧结(Spark Plasma Sintering)所施加的电场强 度 (一般为 $<5 \mathrm{~V} / \mathrm{mm}$ 的直流脉冲电场 $)^{[6-7]}$ 。有些研究 者也尝试使用交流电源或脉冲电源代替闪烧使用的

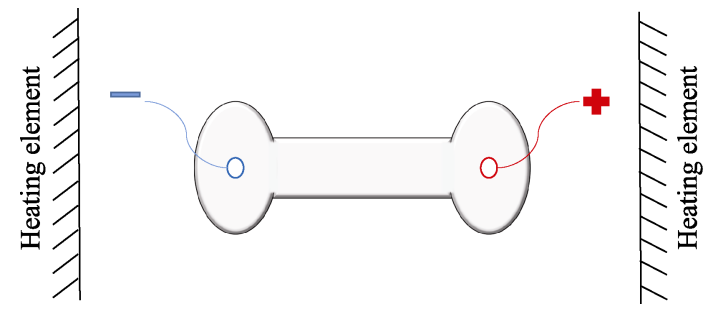

图 1 闪烧装置示意图

Fig. 1 Schematic diagram of flash sintering device 
直流电源 ${ }^{[6-7]}$, 但是它们的烧结机理是否完全相同 还有待进一步研究。对于一些陶瓷材料体系, 控制 烧结气氛也会对闪烧产生影响 ${ }^{[8]}$ 。为了监测闪烧过 程, 还可以接入电子仪表记录电压和电流曲线, 通 过热电偶或红外测温仪探测试样的表面温度, 利用 光学相机或位移计测量试样的线收缩等。由此可见, 闪烧是一种典型的电场辅助无压烧结技术。

根据图 2 所示的闪烧过程的电场强度、电流密 度和功率密度随时间变化曲线, 一般可以将整个过 程分为三个阶段, 分别对应孕育(Incubation)、突变 (Transient)和稳态(Steady-state)过程, 闪烧过程往往 伴随着非线性电导、快速致密化和发光现象 ${ }^{[1-7]}$ 。离 子导体、电子导体、半导体、混合导体, 甚至某些 绝缘体都可以发生闪烧现象 ${ }^{[2-7]}$, 但是不同材料的 致密化行为存在较大差异, 例如, 纯 $\mathrm{SiC}$ 闪烧后并 不能获得高密度 ${ }^{[9]}$; 纯 $\mathrm{ZnO}^{[10]}$ 和掺杂的 $\mathrm{Al}_{2} \mathrm{O}_{3}{ }^{[11]}$ 样 品在闪烧瞬间的线收缩非常有限。因此, 对于不同 材料体系, 是否具有统一的闪烧机理尚无定论。目 前, 氧化钎稳定氧化铅陶瓷是闪烧研究中使用最广 泛且最具代表性的材料体系, 也是闪烧机理研究中 最受关注的材料。此外, 致密的陶瓷样品在合适的 温度和电场作用下也能出现非线性电导和发光现 象 ${ }^{[12]}$ 。冊庸置疑, 闪烧拓展了传统的认知边界, 电 场与物质的相互作用还有许多尚未被认知的机理。

\section{2 闪烧机理的研究进展}

\section{1 闪烧的孕育与引发过程}

在恒温闪烧氧化钎稳定氧化锆陶瓷的孕育阶段, 试样两端加载有恒压直流电场, 通过试样的电流随

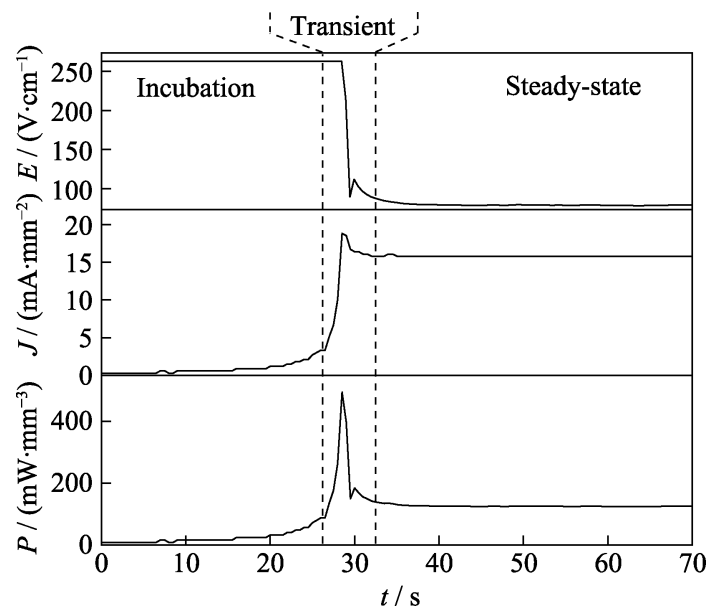

图 2 闪烧过程的电场强度、电流密度和功率密度随时间的 变化曲线

Fig. 2 Electric field strength, current density and power density as a function of time during the flash sintering
着加载时间延长缓慢增加, 如图 2 所示。Gao 等 ${ }^{[13]}$ 对摩尔分数 $3 \%$ 氧化钎稳定氧化锆陶瓷(3YSZ)在闪 烧孕育阶段的电压一电流曲线进行了精确测试, 研 究发现: 当加载电场较小时, 在加载瞬间(约 $0.5 \mathrm{~s}$, 试样温度变化可忽略不计)测得的电压与电流呈典 型的线性关系; 当加载电场超过临界值后, 测得的 电压与电流偏离线性关系, 呈现出明显的非线性特 征。即使考虑焦耳热对试样温度和电导的影响, 3YSZ 试样在高于临界电场条件下测得的电导率也 明显偏大。这说明在闪烧过程中出现的非线性电导 现象与加载的临界电场密切相关。可以认为: 在临界 电场作用下, 内部缺陷反应产生了额外的氧空位 ${ }^{[13-15]}$, 随着孕育时间延长, 内部缺陷反应注入的氧空位数 目增多, 试样的电导率不断升高, 从而使 $3 \mathrm{YSZ}$ 试 样在闪烧过程中出现非线性电导的特征。加载的电 场和试样的初始温度越高, 越有利于产生额外氧空 位, 样品的电导率增加越快。

氧化钇稳定氧化锆陶瓷样品在加载临界电场后 会发生明显的电化学黑化现象(见图 3(a)), 这间接 证明了临界电场作用会提高氧空位浓度。图 3(b)所 示的电子顺磁共振波谱仪(EPR)的测试结果也证明: 闪烧后 3YSZ 样品靠近正极端的氧空位浓度相比闪 烧前有大幅提高。改变闪烧时的氧分压, 闪烧的孕 育过程也会随之发生变化。高氧分压条件下, 需要
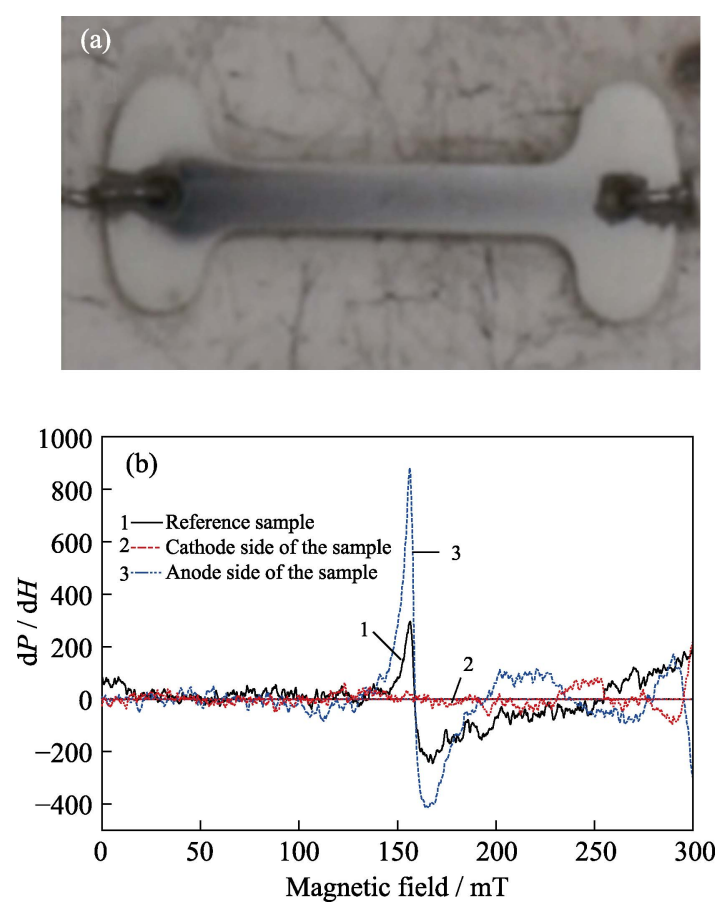

图 3 (a)闪烧氧化钎稳定氧化锆陶瓷样品出现的电化学黑 化现象和(b)闪烧后样品的电子顺磁共振波谱

Fig. 3 (a) Electrochemical blackening of flash sintered $3 \mathrm{YSZ}$ ceramic and (b) EPR result of the flash sintered 3YSZ sample 
更高的温度和更长的孕育时间来引发闪烧, 这说明 氧空位浓度与闪烧孕育过程密切相关 ${ }^{[8]}$ 。由于原位 研究闪烧样品的点缺陷存在巨大的技术挑战, 相关 实验验证还有待进一步探索。

在氧化钎稳定氧化锆陶瓷的闪烧孕育阶段, 可 以观察到靠近负极端先发生电化学黑化。随着孕育 时间延长，黑化区域不断向正极扩展，当黑化区域 到达正极端后, 就会引发闪烧 ${ }^{[16]}$ 。闪烧的引发过程 与氧化锆样品的电导行为密切相关。在孕育阶段, 虽然氧空位浓度大幅增加, 但是试样仍以离子电导 为主。在临界电场的作用下，电子可以从负极进入 氧空位, 并形成色心, 导致氧化锆试样靠近负极端 先发生黑化(见图 3(a))。随着加载时间延长, 被氧空 位捕获的电子数目越来越多, 电子又借助氧空位由 负极逐渐向正极运动，当电子借助色心到达正极后， 瞬间形成电子电导, 使得通过试样的电流迅速升高, 直至限定数值, 从而引发闪烧。Yadav 和 Raj ${ }^{[17]}$ 报导 的电导激活能测试结果表明, 闪烧阶段的电导激活 能小于氧离子的扩散激活能; Jo 和 $\mathrm{Raj}^{[18]}$ 通过电化 学阻抗谱测试也证实了这种由离子电导向电子电导 转变的闪烧引发机制。

Cologna 等 ${ }^{[19]}$ 认为闪烧的引发过程可能与 Frenkel 缺陷的雪崩相关。在临界电场作用下, 氧化 锆陶瓷试样达到 Debye 温度后, 会产生大量的 Frenkel 缺陷, 当缺陷积聚到一定程度, 就会发生缺 陷雪崩, 使电流迅速增大, 引发闪烧。焦耳热失控也 被认为可能是引发闪烧的原因 ${ }^{[20-22]}$, 当积聚的焦耳 热超过样品因对流、辐射和热传导损失的热量时, 就会造成试样温度快速上升, 从而导致试样电导率 增加, 通过试样的电流也快速增大。

\section{2 闪烧阶段的快速致密化}

氧化钎稳定氧化锆陶瓷样品闪烧时, 在几秒钟 内就可以由 $50 \%$ 的相对密度达到几乎完全致密， 大幅缩短烧结时间, 降低能源消耗, 提升烧结效率。 在其它可发生闪烧的陶瓷体系中也可以观察到类似 的快速致密化现象, 这是闪烧引起广泛关注的最主 要原因。闪烧时, 电流直接通过陶瓷试样, 焦耳热的 作用会造成试样的温度上升, 研究人员通过热电偶 测温、红外测温和原位 X 射线衍射(XRD)测试等多 种实验手段对样品闪烧时的实际温度进行了标定, 发现闪烧的致密化速率远远高于试样在相同温度下 的传统烧结过程 ${ }^{[23]}$ 。

在氧化钎稳定氧化锆陶瓷生坏闪烧时, 由于外 加临界电场的作用, 会在试样中产生大量的额外氧
空位，同时带正电的 $V \ddot{\text { o }}$ 会和带负电的 $\mathrm{Y}_{\mathrm{z}_{\mathrm{r}}}{ }^{\prime}$ 向相反的 方向运动造成介电极化, 并在粉体颗粒表面不同位 置产生净电荷。随着临界电场作用时间延长, 粉体 颗粒聚集的表面电荷会越来越多。在闪烧瞬间，相 邻的粉体颗粒由于库仑力的作用发生强烈的相互吸 引, 导致烧结前期的快速致密化 ${ }^{[24]}$ 。这种库仑力产 生的吸引作用, 改变了传统烧结由原子扩散主导的 致密化机理, 使得闪烧过程的烧结激活能远小于传 统烧结过程的激活能(见图 4)。本课题组发现在 $\mathrm{BaTiO}_{3}$ 陶瓷的闪烧过程中, 其烧结前期也存在类似 的作用机理 ${ }^{[25]}$ 。

由于库仑力吸引导致的烧结前期的致密化过程 和表面电荷相关, 因此限制电流的大小会直接影响 样品的密度。限制电流密度越大, 单位截面内的载 流子数目越多, 意味着有更多的表面电荷参与这一 致密化过程。本课题组的实验结果也证明: 3YSZ 试 样闪烧后的密度与限制电流的大小密切相关, 而电 场强度对密度的影响很小 ${ }^{[26]}$ 。需要指出的是, 库仑 力吸引导致的致密化不涉及物质的扩散, 仅在烧结 前期产生影响, 并不能使样品完全致密, 发生库仑 力吸引后, 试样还需在电场作用下通过物质扩散来 进一步排除气孔，提高试样的致密度。

在临界电场作用下，由于产生了大量的氧空位， 闪烧时物质扩散过程也发生了重大改变。本课题组 将纯氧化锆和摩尔分数 $3 \%$ 的氧化钇纳米粉体均匀 混合后再进行闪烧，发现氧化锆由单斜相快速转变 为四方相, 与相同温度下的传统烧结过程相比, 其 反应速率提高了上千倍 ${ }^{[27-28]}$ 。这一过程也清楚地表 明: 在临界电场作用下，氧化钎/氧化锆混合粉体不 仅发生了氧空位的快速运动，也发生了金属阳离子 的快速运动和固溶。临界电场作用不仅对颗粒自身 的表面扩散、晶界扩散和体积扩散有影响，而且对相 邻颗粒间在固相烧结过程中的扩散行为也产生

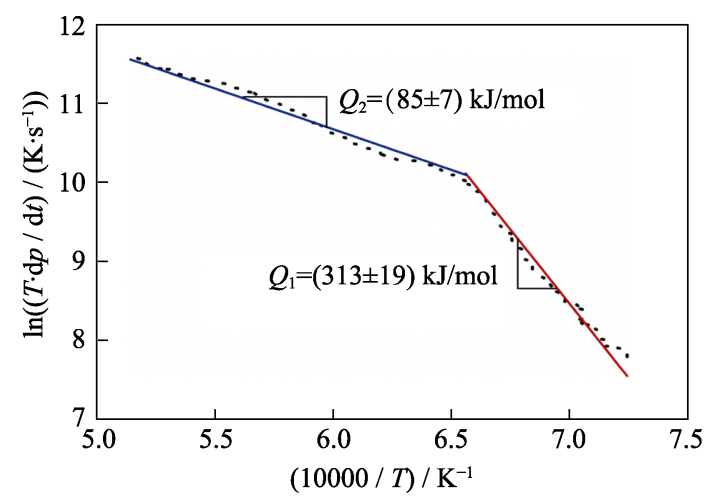

图 43 YSZ 陶瓷闪烧过程中的激活能变化 ${ }^{[24]}$

Fig. 4 Calculated activation energy during flash sintering of $3 \mathrm{YSZ}^{[24]}$ 
了重大影响。闪烧过程的快速致密化可能是氧空位 与金属阳离子形成了缔合缺陷，并且其在临界电场 作用下可以发生快速运动的结果。

Cologna 等 ${ }^{[19]}$ 提出 Frenkel 缺陷在电场作用下发 生雪崩是造成快速致密化的原因。Zhang 等 ${ }^{[10]}$ 和 $\mathrm{Ji}$ 等 ${ }^{[29]}$ 几乎同时提出闪烧瞬间极高的加热速率是造 成快速致密化的根本原因, 并通过快速升温烧结实 验进行了对比验证。除此之外, 还有研究者认为闪 烧瞬间会使晶界处出现局部高温, 形成液相, 从而 提高了致密化速率 ${ }^{[30]}$ 。

\section{3 稳态阶段的晶粒生长与微结构演变}

当氧化钎稳定氧化锆陶瓷发生闪烧之后，电源 会自动由恒压模式切换成恒流模式，使通过试样的 电流保持恒定, 同时由于试样的密度经过闪烧突变 阶段之后得到大幅提升, 试样的电导率也趋于稳定, 闪烧进入稳态阶段。

一般认为闪烧试样在稳态阶段主要受炉温和焦 耳热的双重影响。由于存在对流、辐射和热传导, 试 样芯部的温度会比表面的温度高, 狗骨状试样中心 的温度会比正极和负极两端的温度高, 试样温度沿 电流方向呈对称分布特征。本课题组利用热成像仪 对闪烧试样的表面温度进行了系统研究 ${ }^{[15]}$, 如图 5 所示，试样表面温度呈明显的非对称分布，靠近正 极端的温度比负极端高, 试样表面的最高温度并未 在中心位置, 而是在靠近试样正极的一侧。这说明 在闪烧稳态阶段，电流通过试样除了产生焦耳热， 还会引发内部缺陷反应造成放热或吸热现象，从而 使得试样表面的温度呈现非对称分布的特征 ${ }^{[15]}$ 。当 用交流电场取代直流电场进行闪烧，由于内部缺陷 反应会随电场的变化而改变, 试样表面的温度呈对 称分布，但是不同于单一的焦耳热效应，闪烧试样 中间区域有明显的等温区 ${ }^{[31]}$ 。这也说明临界电场在

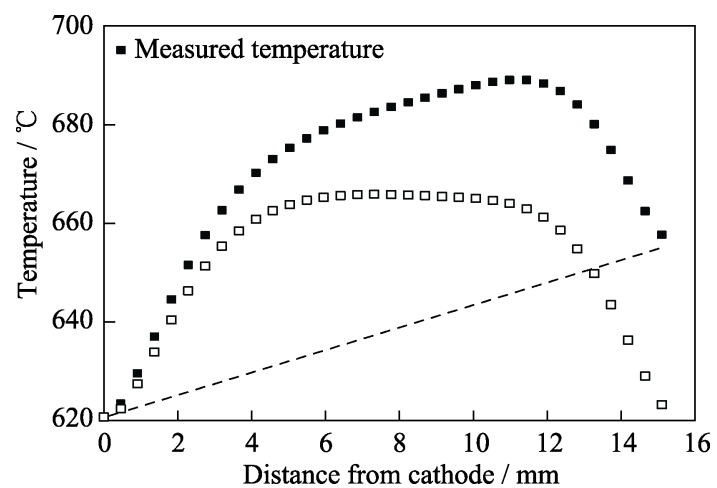

图 $58 \mathrm{YSZ}$ 陶瓷试样表面温度在闪烧稳态阶段随位置的变 化曲线 ${ }^{[15]}$

Fig. 5 Change of the surface temperature of 8YSZ sample as a function of distance from cathode at the steady stage during the flash sintering ${ }^{[15]}$
闪烧过程中会引发内部缺陷反应，从而对试样中的 温度分布和显微结构产生影响。

本课题组在稳态阶段对致密的 3YSZ 试样长时 间保温, 研究了它的晶粒生长过程 ${ }^{[32]}$ 。较大的电流 密度和较长的保温时间会使晶粒明显长大。闪烧试 样中间位置的晶界迁移率相比传统的热处理过程提 高了一倍。如果闪烧过程中只有焦耳热发挥作用, 晶粒尺寸分布应该和焦耳热导致的试样温度分布特 征相对应，即试样中部的温度最高，晶粒尺寸最大; 试样两端的温度最低，晶粒尺寸最小; 焦耳热产生 的温度场沿电流方向呈对称分布还会导致试样中的 晶粒尺寸也呈对称分布。但是, 受试样温度分布和 内部缺陷反应的影响, 闪烧试样的晶粒尺寸分布也 呈现明显的非均匀特征, 正极端的晶粒尺寸远远大 于负极端的晶粒尺寸 ${ }^{[32]}$ 。这说明单一的焦耳热效应 无法解释稳态阶段的晶粒生长现象。在临界电场作 用下, $3 \mathrm{YSZ}$ 中发生的内部缺陷反应会对闪烧样品的 温度分布和晶界结构产生影响 ${ }^{[28,33]}$, 改变界面运动 和晶粒生长过程, 从而使得靠近正极端的晶粒尺寸 大于靠近负极端的晶粒尺寸, 而且试样中部的晶 粒生长速率在内部缺陷反应的作用下得到大幅 提升。

\section{3 闪烧制备新技术研究进展}

\section{1 闪焊技术}

基于闪烧过程中由临界电场所激发的低温快速 传质，本团队发明了同种陶瓷/陶瓷、陶瓷/金属，甚 至异种陶瓷/陶瓷之间快速连接的闪焊技术。利用闪 焊技术，实现了 $3 \mathrm{YSZ}$ 陶瓷和镍基高温合金之间的 连接 ${ }^{[14]}$, 在 $800{ }^{\circ} \mathrm{C}$, 通过施加临界电场, 可以在合 适的限制电流密度下瞬间实现界面的强结合, 结合 强度达到 $133 \mathrm{MPa}$ 。闪焊由内部缺陷反应生成氧空 位和金属镍扩散填充氧空位聚集形成孔洞与裂纹两 个相互竞争的过程来控制，只有在合适的条件下， 孔洞和裂纹形成的速率低于金属镍的填充速率，才 能获得良好的结合强度。由于氧空位在电场作用下 由正极向负极运动, 当反向加载电场时, 会使连接 好的 3YSZ 陶瓷与镍基高温合金快速脱开，因此闪 焊过程主要依赖于临界电场作用下氧化锆陶瓷中出 现的内部缺陷反应。我们还在 3YSZ 陶瓷与钛合金 的闪焊连接中进一步验证了上述机理 ${ }^{[34]}$ 。

如图 6 所示, 3YSZ 陶瓷与 3YSZ 陶瓷之间也可 以通过闪焊技术连接在一起 ${ }^{[35-36]}$ 。在 600 900 ${ }^{\circ} \mathrm{C}$ 的 炉温下, 通过调整电场强度和电流密度, 可以使接 


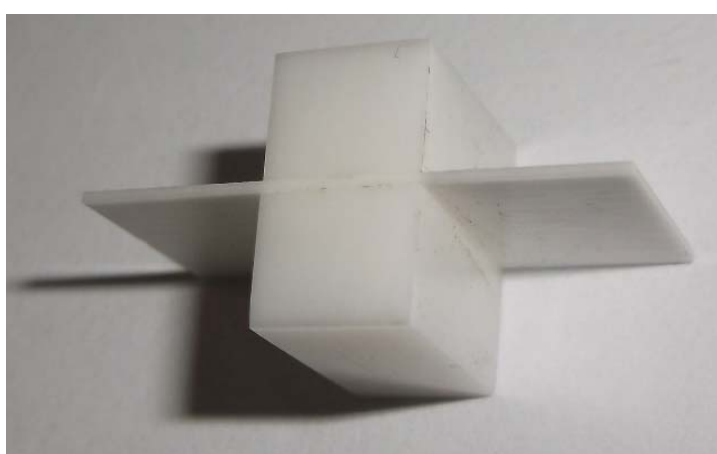

图 6 两个 $3 \mathrm{YSZ}$ 陶瓷块与夹在其中间的 $3 \mathrm{YSZ}$ 陶瓷薄板闪 焊成一个整体构件的照片

Fig. 6 Picture of an integrated component flash joined from two 3 YSZ blocks and a 3 YSZ thin plate

头的三点弯曲强度达到母材强度的 $94 \%$ 99\%，而 且闪焊过程所需的时间非常短, 相比传统扩散焊技 术可以大幅缩短工艺时间。在 3 YSZ 陶瓷与 $3 Y S Z$ 陶瓷的闪焊连接过程中, 除了上述的内部缺陷反应 机理外, 3YSZ 陶瓷在临界电场作用下发生的低温超 塑性变形也会对快速连接产生一定影响。这种内部 缺陷反应导致的闪焊过程还可以用于连接氧化镁掺 杂的氧化铝陶瓷 ${ }^{[37]}$, 以及 $3 Y S Z$ 与氧化铝陶瓷 ${ }^{[38]}$ 。

\section{2 闪烧合成技术}

闪烧不仅能在单相体系中发生, 很多复相体系 也能发生闪烧，例如 $\mathrm{rGO} / 3 \mathrm{YSZ}^{[39]}, 3 \mathrm{YSZ} / \mathrm{Al}_{2} \mathrm{O}_{3}{ }^{[40]}$ 和 $\mathrm{Gd}_{2} \mathrm{O}_{3} / \mathrm{ZrO}_{2}$ [41]等。在氧化锆与氧化钎混合粉体的 闪烧过程中, 氧化钇能发生快速固溶, 进入氧化锆 的晶格, 形成氧化钎稳定的四方相氧化锆 ${ }^{[27-28]}$ 。在 临界电场的作用下, 固溶与相变过程可以在较低的 温度下进行, 而且反应速率极快, 相比传统的高温 处理过程大幅缩短了反应所需时间。

临界电场作用下的低温快速传质实现了金属阳 离子的运动和混合, 因此闪烧还可以用来加速通过 固态反应形成新相的过程。本课题组对 $\mathrm{Al}_{2} \mathrm{O}_{3}$ 和 $\mathrm{Y}_{2} \mathrm{O}_{3}$ 混合粉体进行闪烧, 在低温下实现了它们的快 速反应，获得了 $\mathrm{YAG}$ 陶瓷 ${ }^{[42]}$; 对 $\mathrm{Gd}_{2} \mathrm{O}_{3} 、 \mathrm{Nd}_{2} \mathrm{O}_{3}$ 和 $\mathrm{ZrO}_{2}$ 混合粉体进行闪烧, 在低温下快速合成了具有烧 绿石结构的 $\left(\mathrm{Gd}_{0.2} \mathrm{Nd}_{0.8}\right)_{2} \mathrm{Zr}_{2} \mathrm{O}_{7}$ 陶瓷 ${ }^{[43]}$ 。此外, 本课题 组还通过闪烧技术成功在低温下快速合成了 $\mathrm{BaTiO}_{3}$ 陶瓷 ${ }^{[44]}$ 和固态钠离子电池中的电解质材料 $\mathrm{Na}_{3} \mathrm{Zr}_{2}\left(\mathrm{SiO}_{4}\right)_{2}\left(\mathrm{PO}_{4}\right)^{[45]}$ 。

这种闪烧合成的方法还可以用于制备高摘陶瓷。本 课题组首次在室温条件下实现了五种氧化物的快速固溶, 获得了具有单一岩盐结构的 $\mathrm{Mg}_{0.2} \mathrm{Ni}_{0.2} \mathrm{Co}_{0.2} \mathrm{Cu}_{0.2} \mathrm{Zn}_{0.2} \mathrm{O}$ 高熵陶瓷 ${ }^{[46]}$ 和 $\mathrm{Ca}_{0.2} \mathrm{Co}_{0.2} \mathrm{Ni}_{0.2} \mathrm{Cu}_{0.2} \mathrm{Zn}_{0.2} \mathrm{O}$ 高熵陶瓷 ${ }^{[47]}$ 。 利用相同的原理, 本课题组还实现了钙钛矿结构高 熵 陶 瓷 $\operatorname{Sr}\left(\mathrm{Ti}_{0.2} \mathrm{Y}_{0.2} \mathrm{Zr}_{0.2} \mathrm{Sn}_{0.2} \mathrm{Hf}_{0.2}\right) \mathrm{O}_{3-\mathrm{x}}^{[48]}$ 和
$\left(\mathrm{Bi}_{0.2} \mathrm{Na}_{0.2} \mathrm{~K}_{0.2} \mathrm{Ba}_{0.2} \mathrm{Ca}_{0.2}\right) \mathrm{TiO}_{3}{ }^{[49]}$ 的快速合成。此外, 闪烧合成具有低温快速的特点，可以抑制低熔点氧 化物的挥发，拓宽传统功能陶瓷的成分范围，有望 获得性能更加优异的功能陶瓷。

临界电场作用对界面结构会产生影响, 利用闪 烧的特点, 本课题组最近以 $\mathrm{Al}_{2} \mathrm{O}_{3}$ 和 $\mathrm{Y}_{2} \mathrm{O}_{3}$ 混合粉体 为原料，通过固态烧结获得了具有共晶形貌的 $\mathrm{Al}_{2} \mathrm{O}_{3} / \mathrm{YAG}$ 陶瓷基复合材料(见图 7), 共晶片层的厚 度达到亚微米尺寸, 共晶区域的硬度和断裂韧性可 以与定向凝固技术获得的 $\mathrm{Al}_{2} \mathrm{O}_{3} / \mathrm{YAG}$ 共晶结构相 媲美 ${ }^{[50]}$ 。在此基础之上, 本课题组还通过临界电场 的作用将具有共晶成分的致密多晶 $\mathrm{Al}_{2} \mathrm{O}_{3} / \mathrm{YAG}$ 陶 瓷基复合材料转化成共晶结构, 从而大幅提升材料 的力学性能 ${ }^{[51]}$ 。此外, 通过调控工艺参数, 本课题 组还成功实现了 $\mathrm{Al}_{2} \mathrm{O}_{3} / \mathrm{YAG}$ 共晶结构的细化, 使得 陶瓷材料也可以像金属材料一样通过晶粒细化来提 升力学性能。这种电场辅助下的共晶生长方法在 $\mathrm{Al}_{2} \mathrm{O}_{3} / \mathrm{YSZ}$ 体系中也得到了验证，通过闪烧 $\mathrm{Al}_{2} \mathrm{O}_{3}$ 和 $8 \mathrm{YSZ}$ 混合粉体可以获得具有共晶形貌特征的 $\mathrm{Al}_{2} \mathrm{O}_{3} / \mathrm{YSZ}$ 陶瓷 ${ }^{[52]}$ 。

\section{3 电塑性成形技术}

陶瓷材料由于其固有的脆性使得成型加工非常 困难，限制了它的广泛应用。陶瓷科学家一直希望 能让陶瓷材料获得像金属材料一样的塑性加工能力, 扩大陶瓷材料的应用范围。传统的陶瓷超塑性一般 都是在高温下通过晶界滑移来实现，工艺条件苛刻， 变形速率慢, 实用性不强。

$3 \mathrm{YSZ}$ 陶瓷在闪烧状态下，由于引入了大量的 氧空位缺陷，这些氧空位在临界电场作用下，还能 发生向高维度缺陷(位错和层错等)的转变 ${ }^{[53]}$, 为陶 瓷塑性变形提供了新途径。本课题组已经在较低温 度和极低应力下成功实现了 $3 \mathrm{YSZ}$ 陶瓷的快速拉伸 和弯曲变形(见图 8), 为开展陶瓷塑性成形技术的 研究指出了一个新的发展方向 ${ }^{[54]}$ 。

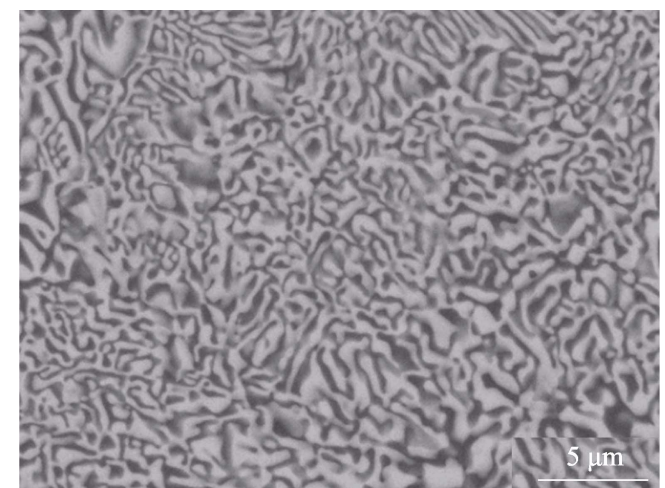

图 7 闪烧形成 $\mathrm{Al}_{2} \mathrm{O}_{3} / \mathrm{YAG}$ 共晶结构的 SEM 照片

Fig.7 SEM image of $\mathrm{Al}_{2} \mathrm{O}_{3} / \mathrm{YAG}$ eutectic structure formed by flash sintering 

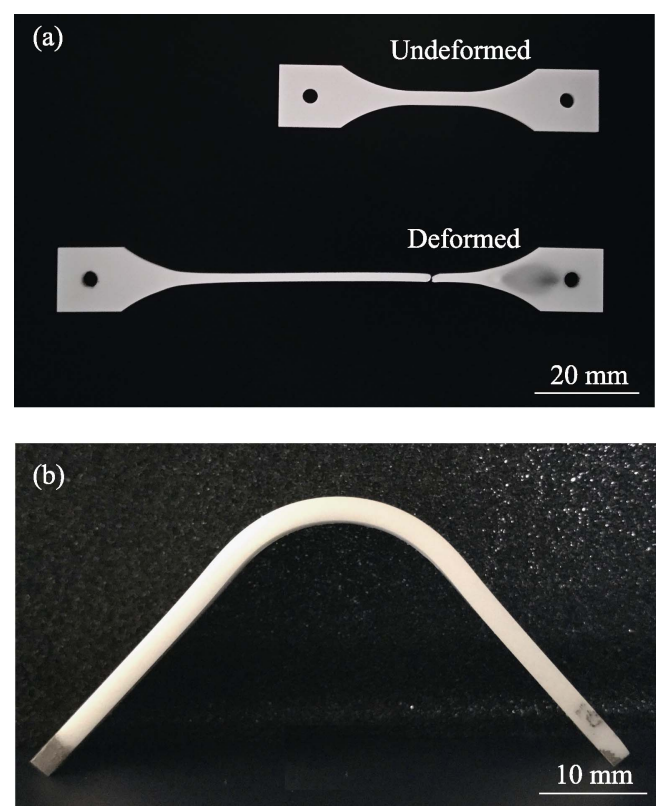

图 8 氧化锆陶瓷在闪烧状态下实现的低温快速(a)拉伸和(b) 弯曲变形

Fig. 8 Fast deformation of 3YSZ under (a) tension and (b) bending test via flash sintering at low temperature

\section{4 结束语}

闪烧作为一种新型的电场辅助烧结技术, 已经 引起了广泛关注。闪烧过程中存在独特的非线性电 导、快速致密化和发光现象, 这在许多材料体系中 都得到了充分验证。目前, 关于闪烧的微观机理还 存在重大分歧，主要包括以下三种观点。

(1)焦耳热效应

闪烧源于电流通过试样引起的焦耳热。随着试 样温度上升, 试样的电阻不断降低, 使得电流迅速 增大，最终导致焦耳热失控，引发闪烧。同时，焦耳 热造成的快速升温可以加速致密化过程, 提升烧结 效率。闪烧样品的发光现象则归因于高温导致的热 辐射发光。目前, 正在迅猛发展的超快高温烧结技 术正是源于这种认识 ${ }^{[55]}$ 。

\section{(2)Frenkel 缺陷}

Jongmanns 等 ${ }^{[56]}$ 提出在临界电场作用下，当试 样温度高于 Debye 温度后, 临近布里渊区边缘的晶 格振动被激发, 如果这种晶格振动的增殖速率达到 足够高时, 就能在样品中产生大量的 Frenkel 缺陷 对。经过一定的孕育阶段后, Frenkel 缺陷的雪崩会 使闪烧样品发生电导突变, 同时造成快速传质, 实 现致密化。闪烧过程中出现的发光现象是由电子和 空穴弥合造成的电致发光 ${ }^{[12]}$ 。

\section{(3)氧空位缺陷}

氧化锆陶瓷闪烧时, 在样品两端施加了临界电 场, 试样内部会发生电化学缺陷反应, 形成远高于
热力学平衡状态的氧空位浓度, 电子被氧空位束缚 后产生色心，使得样品出现黑化现象，黑化区域由 负极向正极扩展, 样品由离子电导转变为电子电导, 从而产生电导突变。颗粒表面的介电极化和电荷聚 集会产生库仑力吸引，从而提高烧结初期的致密化 速率。氧空位与金属阳离子形成的缔合缺陷在临界 电场作用下的快速运动可能是闪烧产生快速致密化 的重要原因。

闪烧瞬间的持续时间一般只有 1 2 s, 给实验研 究带来了巨大的挑战, 目前提出的微观机理都尚未 获得实验证实，未来还需结合先进的分析检测技术， 进一步对闪烧机理展开深入系统的研究。

焦耳热在闪烧过程中发挥了重要作用, 这是冊 庸置疑的事实, 对样品形状、电极形状、电极类型、 升降温速率等进行合理设计, 有望通过电场辅助烧 结技术获得致密均匀的样品, 实现陶瓷材料的快速 高效烧结 ${ }^{[57]}$, 这将对小尺寸 ( 厘米级)陶瓷样品的 制备产生颠覆性的影响 ${ }^{[5]}$, 同时也会对放电等离子 烧结技术的工业应用产生促进作用。

闪烧过程中产生的非焦耳热效应更值得关注 ${ }^{[58]}$ 。 本团队在自然科学基金重点项目支持下开展的陶瓷 闪焊技术、闪烧合成技术和电塑性成形技术的研究 已经充分证明：电场作用下产生的非焦耳热效应将 对陶瓷制备技术产生积极作用。加强对非焦耳热效 应微观机理的研究, 可对材料缺陷的认识达到新的 高度, 对材料科学的发展会产生重大影响 ${ }^{[99]}$ 。利用闪 烧过程产生的非焦耳热效应还可以创新陶瓷材料

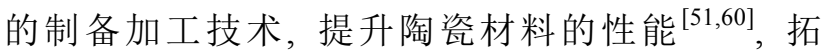
宽陶瓷材料的应用范围, 对陶瓷工业的发展有重要 意义。

\section{参考文献:}

[1] COLOGNA M, RASHKOVA B, RAJ R. Flash sintering of nanograin zirconia in $<5 \mathrm{~s}$ at $850{ }^{\circ} \mathrm{C}$. J. Am. Ceram. Soc., 2010, 93 (11): 3556-3559.

[2] REN K, LIU J, WANG Y. Flash sintering of yttria-stabilized zirconia: fundamental understanding and applications. Scripta Mater., 2020, 187: $371-378$

[3] 傅正义, 季伟, 王为民. 陶瓷材料闪烧技术研究进展. 硅酸盐学 报, 2017, 45(9): 1211-1219.

[4] 谢志鹏, 许靖壁, 安迪. 先进陶瓷材料烧结新技术研究进展. 中 国材料进展, 2019, 38(9): 821-830.

[5] 苏兴华, 吴亚娟, 安盖, 等. 陶瓷材料闪烧机理研究进展. 硅酸 盐学报, 2020, 48(12): 1872-1879.

[6] YU M, GRASSO S, MCKINNON R, et al. Review of flash sintering: materials, mechanisms and modelling. Adv. Appl. Ceram., 2017, 116(1): 24-60.

[7] BIESUZ M, SGLAVO V M. Flash sintering of ceramics. J. Eur. Ceram. Soc., 2019, 39: 115-143.

[8] LIU D, CAO Y, LIU J, et al. Effect of oxygen partial pressure on temperature for onset of flash sintering 3YSZ. J. Eur. Ceram. Soc., 2018, 38: 817-820.

[9] ZAPATA-SOLVAS E, BONILlA S, WILSHAW P R, et al. Preliminary investigation of flash sintering of SiC. J. Eur. Ceram. Soc., 2013, 33: 2811-2816. 
[10] ZHANG Y, NIE J, CHAN J M, et al. Probing the densification mechanisms during flash sintering of ZnO. Acta Mater., 2017, 125: $465-475$.

[11] COLOGNA M, FRANCIS J S C, RAJ R. Field assisted and flash sintering of alumina and its relationship to conductivity and $\mathrm{MgO}$ doping. J. Eur. Ceram. Soc., 2011, 31: 2827-2837.

[12] LEBRUN J M, RAJ R. A first report of photoemission in experiments related to flash sintering. J. Am. Ceram. Soc., 2014, 97(8): 2427-2430.

[13] GAO Y, LIU F, LIU D. Electrical-field induced nonlinear conductive behavior in dense zirconia ceramic. J. Mater. Sci. Technol., 2017, 33: 897-900

[14] XIA J, REN K, WANG Y, et al. Reversible flash-bonding of zirconia and nickel alloys. Scripta Mater., 2018, 153: 31-34.

[15] LIU G. LIU D, LIU J, et al. Asymmetric temperature distribution during steady stage of flash sintering dense zirconia. J. Eur. Ceram. Soc., 2018, 38: 2893-2896.

[16] LIU J, LIU D, WANG Y, et al. The Onset of Flash Sintering 8YSZ. ECI Conference on Electric Field Enhanced Processing of Advanced Materials II: Complexities and Opportunities, Tomar, Portugal, March 10-15, 2019.

[17] YADAV D, RAJ R. The onset of the flash transition in single crystals of cubic zirconia as a function of electric field and temperature. Scripta Mater., 2017, 134: 123-127.

[18] JO S, RAJ R. Transition to electronic conduction at the onset of flash in cubic zirconia. Scripta Mater., 2020, 174: 29-32.

[19] COLOGNA M, PRETTE A L G, RAJ R. Flash-sintering of cubic yttria-stabilized zirconia at $750{ }^{\circ} \mathrm{C}$ for possible use in SOFC manufacturing. J. Am. Ceram. Soc., 2011, 94(2): 316-319.

[20] DONG Y, CHEN I W. Predicting the onset of flash sintering. J. Am. Ceram. Soc., 2015, 98(8): 2333-2335.

[21] ZHANG Y, JUNG J, LUO J. Thermal runaway, flash sintering and asymmetrical microstructural development of $\mathrm{ZnO}$ and $\mathrm{ZnO}-\mathrm{Bi}_{2} \mathrm{O}_{3}$ under direct currents. Acta Mater., 2015, 94: 87-100.

[22] TODD R I, ZAPATA-SOLVAS E, BONILLA R S. Electrical characteristics of flash sintering: thermal runaway of Joule heating. J. Eur. Ceram. Soc., 2015, 35: 1865-1877.

[23] FRANCIS J S C, RAJ R. Flash-sinterforging of nanograin zirconia: field assisted sintering and superplasticity. J. Am. Ceram. Soc., 2012, 95(1): 138-146.

[24] REN K, WANG Q, LIAN Y, et al. Densification kinetics of flash sintered $3 \mathrm{~mol} \% \mathrm{Y}_{2} \mathrm{O}_{3}$ stabilized zirconia. J. Alloys Compd., 2018, 747: $1073-1077$.

[25] REN K, HUANG S, CAO Y, et al. The densification behavior of flash sintered $\mathrm{BaTiO}_{3}$. Scripta Mater., 2020, 186: 362-365.

[26] LIU D, LI X, LIU F, et al. Effect of the current density on the densification of $3 \mathrm{~mol} \%$ yttria-stabilized zirconia in flash sintering. J. Alloys Compd., 2020, 825: 154061.

[27] LIU J, LIU D, WANG Y, et al. Flash Sintering Yttria-stablized Zirconia (3Y-TZP) and Zirconia-3mol\%yttria Nanocomposites. $39^{\text {th }}$ International Conference and Exposition on Advanced Ceramics and Composites, Daytona Beach, USA, January 25-30, 2015.

[28] LIU D, GAO Y, LIU J, et al. Effect of holding time on the microstructure and properties of flash sintered $\mathrm{Y}_{2} \mathrm{O}_{3}$-doped $\mathrm{ZrO}_{2}$. Ceram. Int., 2016, 42: 17442-17446.

[29] JI W, PARKER B, FALCO S, et al. Ultra-fast firing: effect of heating rate on sintering of $3 \mathrm{YSZ}$, with and without an electric field, J. Eur. Ceram. Soc., 2017, 37: 2547-2551.

[30] NARAYAN J. A new mechanism for field-assisted processing and flash sintering of materials. Scripta Mater., 2013, 69: 107-111.

[31] ZHU F, PENG X, LIU J, et al. Surface temperature distribution on dense $8 \mathrm{YSZ}$ ceramics during the steady stage in AC flash sintering. Ceram. Int., 2021, 47: 2884-2887.

[32] REN K, XIA J, WANG Y. Grain growth kinetics of $3 \mathrm{~mol} \%$ yttria-stabilized zirconia during flash sintering. J. Eur. Ceram. Soc., 2019, 39: 1366-1373.

[33] LIU D, LIU J, GAO Y, et al. Effect of the applied electric field on the microstructure and electrical properties of flash-sintered 3YSZ ceramics. Ceram. Int., 2016, 42: 19075-19079.

[34] XIA J, REN K, WANG Y. Reversible joining of zirconia to titanium alloy. Ceram. Int., 2019, 45: 2509-2515.

[35] XIA J, REN K, WANG Y. One-second flash joining of zirconia ceramic by an electric field at low temperatures. Scripta Mater.,
2019, 165: 34-38

[36] XIA J, REN K, LIU W, et al. Ultrafast joining of zirconia ceramics using electric field at low temperatures. J. Eur. Ceram. Soc., 2019, 39: 3173-3179.

[37] XIA J, REN K, WANG Y. Flash joining of alumina ceramics under a small current density. J. Eur. Ceram. Soc., 2021, 41: 2782-2789.

[38] XIA J, REN K, WANG Y. Rapid joining of heterogeneous ceramics with a composite interlayer under the action of an electric field. J. Eur. Ceram. Soc., 2021, 41: 7164-7169.

[39] XIAO W, NI N, FAN X, et al. Ambient flash sintering of reduced graphene oxide/zirconia composites: role of reduced graphene oxide, J. Mater. Sci. Technol., 2021, 60: 70-76.

[40] JIA Y, SU X, WU Y, et al. Flash sintering of $3 \mathrm{YSZ} / \mathrm{Al}_{2} \mathrm{O}_{3}$-platelet composites. J. Am. Ceram. Soc., 2020, 103: 2351-2361.

[41] XU C, WANG L, BAI B, et al. Rapid synthesis of $\mathrm{Gd}_{2} \mathrm{Zr}_{2} \mathrm{O}_{7}$ ceramics by flash sintering and its aqueous durability. J. Eur. Ceram. Soc., 2020, 40: 1620-1625.

[42] ZHANG H, WANG Y, LIU J, et al. Reaction assisted flash sintering of $\mathrm{Al}_{2} \mathrm{O}_{3}$-YAG ceramic composites with eutectic composition. Ceram. Int., 2019, 45: 13551-13555.

[43] LIAN Y, REN K, WANG Q, et al. Rapid immobilization of simulated radionuclide $\mathrm{Nd}$ at low temperatures by flash reaction. Ceram. Int., 2019, 45: 22388-22393.

[44] ZHU Y, MA B, WANG K, et al. Electric field-assisted solid-state reaction of $\mathrm{BaCO}_{3}-\mathrm{TiO}_{2}$ system. J. Am. Ceram. Soc., 2021, 104: 6572-6578.

[45] REN K, CAO Y, CHEN Y, et al. Flash sintering of $\mathrm{Na}_{3} \mathrm{Zr}_{2}\left(\mathrm{SiO}_{4}\right)_{2}$ $\left(\mathrm{PO}_{4}\right)$ solid-state electrolyte at furnace temperature of $700{ }^{\circ} \mathrm{C}$. Scripta Mater., 2020, 187: 384-389.

[46] LIU D, PENG X, LIU J, et al. Ultrafast synthesis of entropystabilized oxide at room temperature. J. Eur. Ceram. Soc., 2020, 40: 2504-2508.

[47] LI W, CHEN L, LIU D, et al. Ultra-low temperature reactive flash sintering synthesis of high-enthalpy and high-entropy $\mathrm{Ca}_{0.2} \mathrm{Co}_{0.2}$ $\mathrm{Ni}_{0.2} \mathrm{Cu}_{0.2} \mathrm{Zn}_{0.2} \mathrm{O}$ oxide ceramics. Mater. Lett., 2021, 304: 130679.

[48] WANG K, MA B, LI T, et al. Fabrication of high-entropy perovskite oxide by reactive flash sintering. Ceram. Int., 2020, 46: 18358-18361.

[49] LIU J, REN K, MA C, et al. Dielectric and energy storage properties of flash-sintered high-entropy $\left(\mathrm{Bi}_{0.2} \mathrm{Na}_{0.2} \mathrm{~K}_{0.2} \mathrm{Ba}_{0.2} \mathrm{Ca}_{0.2}\right) \mathrm{TiO}_{3}$ ceramic. Ceram. Int., 2020, 46: 20576-20581.

[50] LIU J, XU X, LIU D, et al. Ultrafast formation of $\mathrm{Al}_{2} \mathrm{O}_{3}-\mathrm{Y}_{3} \mathrm{Al}_{5} \mathrm{O}_{12}$ eutectic ceramic by flash sintering. J. Am. Ceram. Soc., 2020, 103: 4051-4056.

[51] XU X, FAN J, LIU J, et al. Formation of eutectic structure in dense $\mathrm{Al}_{2} \mathrm{O}_{3}-\mathrm{YAG}$ composite by electric field treatment. Ceram. Int., 2021, 47: 23647-23652.

[52] YAO S, LIU D, LIU J, et al. Ultrafast preparation of $\mathrm{Al}_{2} \mathrm{O}_{3}-\mathrm{ZrO}_{2}$ multiphase ceramics with eutectic morphology via flash sintering. Ceram. Int., 2021, 47: 31555-31560.

[53] LU S, LIU J, SHAO G, et al. On the electric conduction of $\mathrm{ZrO}_{2}$ in the steady stage of flash sintering. Ceram. Int., 2020, 46: 5715-5718.

[54] LIU D, LIU J, WANG Y, et al. DC Electric Field Assisted 3YSZ Ceramic Superplastic Deformation. ECI Conference on Electric Field Enhanced Processing of Advanced Materials II: Complexities and Opportunities, Tomar, Portugal, March 10-15, 2019.

[55] WANG C, PING W, BAI Q, et al. A general method to synthesize and sinter bulk ceramics in seconds. Science, 2020, 368: 521-526.

[56] JONGMANNS M, RAJ R, WOLF D E. Generation of Frenkel defects above the Debye temperature by proliferation of phonons near the Brillouin zone edge. New J. Phys., 2018, 20: 093013.

[57] JONES G M, BIESUZ M, JI W, et al. Promoting microstructural homogeneity during flash sintering of ceramics through thermal management. MRS Bull., 2021, 46: 59-66.

[58] GUillon O, DE SOUZA R A, MiShRA $\mathrm{T}$, et al. Electric-field-assisted processing of ceramics: nonthermal effects and related mechanisms. MRS Bull., 2021, 46: 52-58.

[59] RAJ R. KULKARNI A, LEBRUN J M, et al. Flash sintering: a new frontier in defect physics and materials science. MRS Bull., 2021, 46: 36-43.

[60] MA B, ZHU Y, WANG K, et al. Microstructure and dielectric property of flash sintered $\mathrm{SiO}_{2}$-coated $\mathrm{BaTiO}_{3}$ ceramics. Scripta Mater., 2019, 170: 1-5. 